CENTRO UNIVERSITÁRIO DE BRASÍLIA - UniCEUB

FACULDADE DE CIÊNCIAS DA EDUCAÇÃO E DA SAÚDE - FACES

FACULDADE DE ENFERMAGEM

PROGRAMA DE INICIAÇÃO CIENTÍFICA

FERNANDA SOUZA LOPES

CONHECIMENTO DE ESTUDANTES UNIVERSITÁRIOS SOBRE A CIRURGIA BARIÁTRICA (CB) 


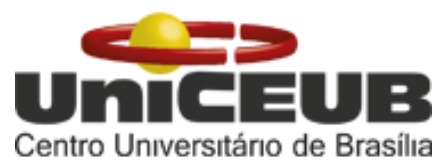

FERNANDA SOUZA LOPES

CONHECIMENTO DE ESTUDANTES UNIVERSITÁRIOS SOBRE A CIRURGIA BARIÁTRICA (CB)

Relatório final de pesquisa de Iniciação Científica
apresentado à Assessoria de Pós-Graduação e
Pesquisa pela Faculdade de Enfermagem da
Faculdade de Ciências da Educação e da Saúde
- FACES do Centro Universitário de Brasília
(UniCeub).

Orientação: Linconl Agudo Oliveira Benito. 


\title{
CONHECIMENTO DE ESTUDANTES UNIVERSITÁRIOS SOBRE A CIRURGIA BARIÁTRICA (CB)
}

Fernanda Souza Lopes - UniCEUB, PIC Institucional, aluno bolsista, fernandaslopes1@hotmail.com

\author{
Linconl Agudo Oliveira Benito - UniCEUB, professor orientador, \\ linconlbenito@yahoo.com.br
}

A cirurgia bariátrica $(\mathrm{CB})$ se constitui enquanto método eficiente no tratamento à obesidade (CID 10 - E66) e suas comorbidades, permitindo a perca de peso e regulação de vários parâmetros relacionados ao funcionamento corporal. Trata-se de um estudo transversal e de abordagem quantitativa que analisou o conhecimento de estudantes de uma instituição de ensino superior (IES) com sede em Brasília (D.F.) sobre a CB. Para aquisição dos subsídios necessários a edificação do presente estudo, foi utilizado um instrumento de coleta de dados (ICD) proposto por Garrido Jr (2004), possuidor do título "Teste de entendimento aos pacientes candidatos à cirurgia da obesidade mórbida (TEPCCOM)", permitindo desta forma aferir o conhecimento dos atores sociais participantes. Foram também utilizados artigos de periódicos científicos, produções acadêmicas e legislação correlata, adquiridas após buscas bibliográficas eletrônicas junto à base de dados informatizados nacionais e internacionais (CUIDEN, LILACS, MEDLINE, MINERVAUFRJ, SABER-USP, TESES-FIOCRUZ). O presente estudo foi submetido a avaliação e tratamento bioético, sendo o mesmo aprovado pelo Comitê de Ética e Pesquisa do Centro Universitário de Brasília (CEP/UNICEUB), com o número da CAAE "50679015.6.0000.0023". Foram entrevistados quatrocentos (400) estudantes, sendo que $48 \%(n=192)$ cursavam "enfermagem", $24 \%(n=96)$ cursavam "nutrição", $15,50 \%$ ( $n=62)$ cursavam "medicina", $8,25 \%(n=33)$ cursavam "biomedicina" e 4,25\% $(n=17)$ cursavam "biologia". O perfil dos atores sociais participantes do presente estudo se constituiu de 78,75\% ( $n=315)$ pessoas do sexo feminino, $87,75 \%(n=351)$ solteiras, $70,75 \%(n=283)$ não desenvolvem atividade remunerada, 93,25\% $(n=373)$ negam enfermidade(s), 94,50\% ( $n=378)$ negam tabagismo, 78\% ( $n=312)$ negam consumo medicamentoso, 93\% ( $n=372)$ negam etilismo, 99,25\% ( $n=397)$ negam hipertensão arterial $(H A), 100 \%(n=400)$ negam diabetes mellitus (DM), 81,50\% $(n=326)$ negam sobrepeso e $95 \%(n=380)$ negam obesidade. Por meio do presente estudo foi possível verificar que os graduandos em saúde possuem conhecimento em relação a CB. Foi identificado ainda reduzido conhecimento dentre os entrevistados, apenas na categoria analítica relacionada a necessidade de suplementação alimentar utilizando polivitamínicos após a realização da CB.

Palavras-chave: Conhecimento. Estudantes. Obesidade. Obesidade mórbida. Cirurgia bariátrica. 


\section{KNOWLEDGE STUDENTS OF UNIVERSITY OF SURGERY BARIATRIC (SB)}

Bariatric surgery (CB) is constituted as an efficient method in the treatment of obesity (ICD-10 - E66) and its comorbidities, allowing for weight reduction and regulation of various parameters related to body function. This is a cross-sectional study and a quantitative approach that examined the knowledge of students from a higher education institution (HEI) with headquarters in Brasilia (DF) about the CB. To acquire the necessary support to building the present study, we used a data collection instrument (DCI) proposed by Garrido Jr (2004), title holder "test of understanding to patients eligible for surgery of morbid obesity (TEPCCOM)" thereby allowing assess knowledge of participants stakeholders. Were also used articles of scientific journals, academic productions and related legislation, acquired after electronic literature searches at the base of national and international computerized data (CUIDEN, LILACS, MEDLINE, MINERVA-UFRJ, SABER-USP, ThesisFIOCRUZ). This study was submitted for assessment and bioethical treatment, and the same approved by the Research Ethics Committee of the University Center of Brasilia (CEP/UNICEUB), with the CAAE number "50679015.6.0000.0023". They were four hundred respondents (400) students, and $48 \%(n=192)$ were enrolled in "nursing", 24\% ( $n=96)$ were enrolled in "nutrition", 15.50\% $(n=62)$ were enrolled in "medicine", 8,25\% ( $n=33)$ were enrolled in "biomedicine" and 4.25\% ( $n=17)$ were enrolled in "biology". The profile of the social actors in the present study consisted of $78.75 \%(n=315)$ females, $87.75 \%(n=351)$ single, $70.75 \%(n=283)$ do not develop remunerated activity, 93.25\% $(n=373)$ denied disease, $94.50 \% \quad(n=378)$ deny smoking, $78 \% \quad(n=312)$ deny drug consumption, 93\% $(n=372)$ deny alcohol consumption, 99 25\% $(n=397)$ deny arterial hypertension $(A H), 100 \%(n=400)$ deny diabetes mellitus (DM), 81.50\% ( $n=326)$ deny overweight and 95\% $(n=380)$ deny obesity. Through this study we found that the health graduate students have knowledge about the CB. It has been identified yet little knowledge among the respondents, only in the analytic category related the need for food supplementation using multivitamins after completion of the CB.

Key-words: Knowledge. Students. Obesity. Morbid obesity. Bariatric surgery. 


\section{CONOCIMENTO DE ESTUDIANTES DE LA UNIVERSIDAD SOBRE CIRURGIA BARIÁTRICA}

La cirugía bariátrica $(\mathrm{CB})$ está constituido como un método eficaz en el tratamiento de la obesidad (CIE-10 - E66) y sus comorbilidades, lo que permite la reducción de peso y la regulación de diversos parámetros relacionados con la función del cuerpo. Se trata de un estudio transversal y un enfoque cuantitativo que examina el conocimiento de los estudiantes de una institución de educación superior (IES), con sede en Brasilia (DF) sobre la CB. Para adquirir el apoyo necesario para construir el presente estudio, se utilizó un instrumento de recolección de datos (DCl) propuestas por Garrido Jr (2004), poseedor del título "prueba de comprensión a los pacientes que pueden beneficiarse de la cirugía de la obesidad mórbida (TEPCCOM)" permitiendo con ello que los interesados evaluar los conocimientos de los participantes. También había sido utilizados artículos de revistas científicas, producciones académicas y la legislación relacionada, adquiridos después de las búsquedas electrónicas de literatura en la base de datos informatizados nacionales e internacionales (CUIDEN, LILACS, MEDLINE, MINERVA-UFRJ, SABER-USP, TESIS-FIOCRUZ). Este estudio fue presentado para su evaluación y tratamiento de la bioética, y la misma aprobado por el Comité Ético de Investigación del Centro Universitário de Brasília (CEP/UNICEUB), con el número CAAE "50679015.6.0000.0023". Fueron entrevistados cuatrocientos (400) estudiantes, y el $48 \%(n=192)$ fueron incluidos en "enfermería", el $24 \%(n=96)$ se inscribieron en la "nutrición", $15,50 \% \quad(n=62)$ se inscribieron en la "medicina", $25 \% \quad(n=33)$ se inscribieron en la "biomedicina" y 4,25\% ( $n=17)$ se inscribieron en la "biología". El perfil de los actores sociales en el presente estudio consistió en $78,75 \% \quad(n=315)$ mujeres, $87,75 \% \quad(n=351)$, solo $70,75 \% \quad(n=283)$ no desarrollan actividad remunerada, 93,25\% ( $n=373)$ nega la enfermedad(s), 94,50\% $(n=378)$ nega el tabaquismo, $78 \%(n=312)$ nega el consumo de drogas, 93\% $(n=372)$ nega el consumo de alcohol, 99,25\% ( $n=397)$ nega la hipertensión arterial (HTA), 100\% $(n=400)$ nega la diabetes mellitus (DM), $81,50 \%(n=326)$ nega el sobrepeso y $95 \%$ $(n=380)$ nega la obesidad. A través de este estudio hemos encontrado que los estudiantes con especialización en salud tienen conocimiento acerca de la CB. Se ha identificado todavía poco conocimiento entre los encuestados, sólo en la categoría analítica relacionada con la necesidad de suplementación alimentaria utilizando multivitaminas después de la finalización de la CB.

Palabras clave: Conocimiento. Estudiantes. La obesidad. La obesidad mórbida. Cirugía bariátrica. 
SUMÁRIO:

\begin{tabular}{|c|c|c|}
\hline № & Itens do projeto de pesquisa: & Pág. \\
\hline & Сара & 01 \\
\hline & Capa de rosto & \\
\hline & Resumo & 03 \\
\hline & 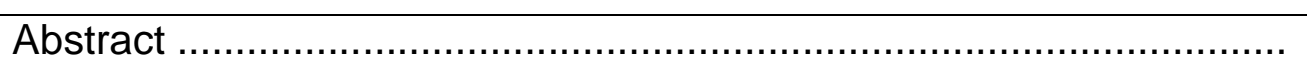 & 04 \\
\hline & 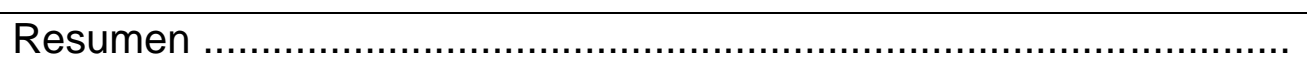 & 05 \\
\hline & 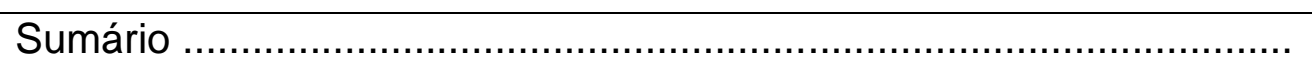 & 06 \\
\hline & 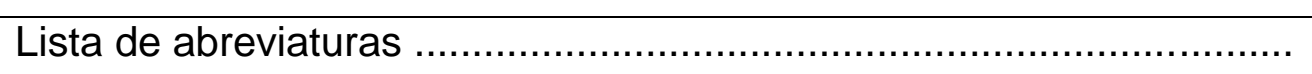 & 07 \\
\hline & Lista de tabelas & 08 \\
\hline & 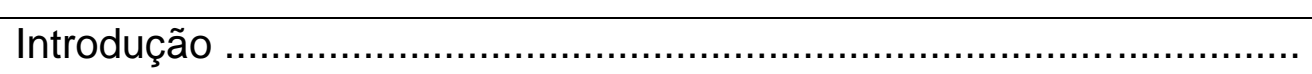 & 09 \\
\hline & Objetivos da pesquisa & \\
\hline & 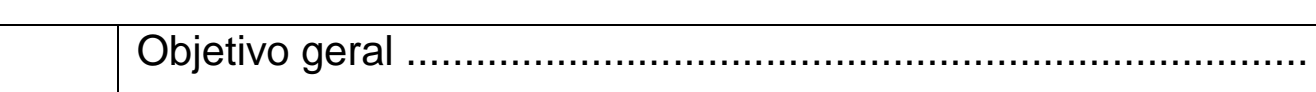 & \\
\hline & 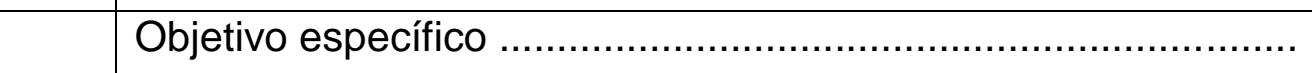 & 13 \\
\hline & 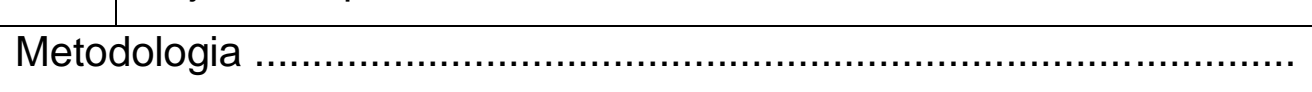 & 13 \\
\hline & 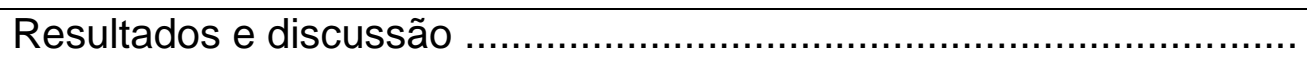 & \\
\hline & 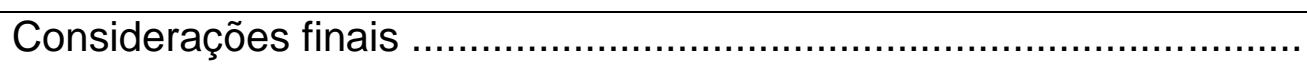 & 22 \\
\hline & 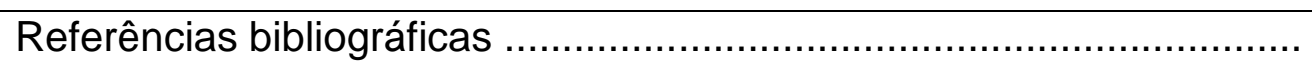 & 23 \\
\hline & Anexos da pesquisa & 27 \\
\hline & 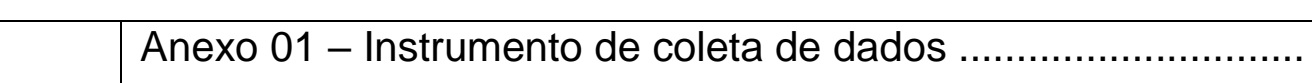 & 27 \\
\hline & \begin{tabular}{l|lcccc} 
Anexo 02 & - & Comprovante de submissão do projeto de \\
pesquisa ao & Comitê & de Ética e & Pesquisa do Centro \\
Universitário & de & Brasília & (CEP-UNICEUB)
\end{tabular} & 29 \\
\hline & 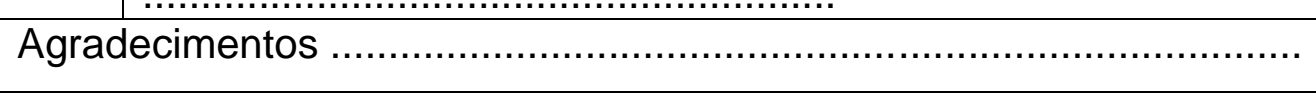 & \\
\hline
\end{tabular}




\section{LISTA DE ABREVIATURAS:}

BGYR: By-pass gástrico em Y-de-Roux (Tipo de cirurgia bariátrica).

BVS: Biblioteca Virtual em Saúde.

Ca: Cálcio (Elemento químico).

CA: Câncer (enfermidade).

CB: Cirurgia bariátrica (Modalidade cirúrgica).

CEP: Comitê de Ética e Pesquisa.

CNS: Conselho Nacional de Saúde.

DBP/DS: Derivação biopancreática/ Duodenal Switch (Tipo de cirurgia bariátrica).

DeCS: Descritores em Ciências da Saúde.

DF: Distrito Federal.

DGYR: Derivação Gástrica em Y de Roux (tipo de cirurgia bariátrica).

DM: Diabetes mellitus (enfermidade).

DM2: Diabetes mellitus tipo 2 (enfermidade).

EUA: Estados Unidos da América (Nação).

Fe: Ferro (elemento químico).

HAS: Hipertensão arterial sistêmica (enfermidade).

HVW: Hipertrofia ventricular esquerda (enfermidade).

IBGE: Instituto Brasileiro de Geografia e Estatística (Órgão estatístico nacional).

ICD: Instrumento de coleta de dados (questionário).

IES: Instituição de ensino superior.

IMC: Índice de massa corpórea (Medida ou parâmetro de avaliação corporal).

kg/m²: Kilograma por metro quadrado (Medida ou parâmetro de avaliação corporal).

MS: Ministério da Saúde (Órgão máximo de gestão da saúde no Brasil).

OMS: Organização Mundial da Saúde.

PO: Pós-operatório (Tempo cirúrgico).

SAOS: Síndrome da apneia obstrutiva do sono (enfermidade).

SBCBM: Sociedade Brasileira de Cirurgia Bariátrica e Metabólica.

SM: Síndrome metabólica (enfermidade).

TCLE: Termo de Consentimento Livre e Esclarecido.

Z: Zinco (Elemento químico). 
LISTA DE TABELAS:

\begin{tabular}{|c|c|}
\hline & Pág. \\
\hline $\begin{array}{l}\text { Tabela } 01 \text { - Frequência de cirurgias bariátricas realizadas no Brasil, entre } \\
\text { os anos de } 2003 \text { à } 2012, \text { por fonte financiadora }\end{array}$ & 10 \\
\hline $\begin{array}{l}\text { Tabela } 02 \text { - Perfil socioeconômico e demográfico dos atores sociais } \\
\text { participantes do presente estudo, por categorias analíticas, Brasília, Distrito } \\
\text { Federal (D.F.), }(\mathrm{n}=400)\end{array}$ & 15 \\
\hline 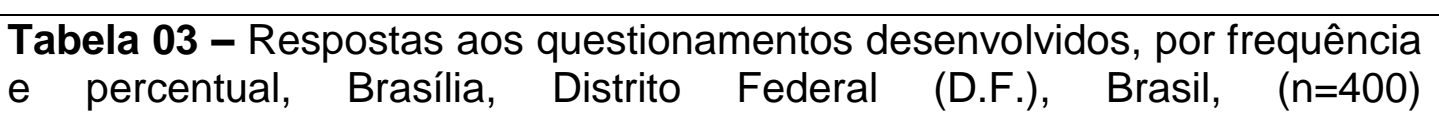 & 16 \\
\hline
\end{tabular}




\section{INTRODUÇÃO:}

Segundo a Organização Mundial de Saúde (OMS), a obesidade é uma condição caracterizada por anormal ou excessivo acúmulo de gordura em tecidos adiposos com vários prejuízos para a saúde dos indivíduos afetados (OMS, 2000). Nesse sentido, esse problema de saúde é considerado uma enfermidade crônica e de complexo controle que, em suas representações mais graves, poderá permitir o aparecimento de complicadores e insucessos terapêuticos (OMS, 2009).

A obesidade é uma doença que afeta as nações desenvolvidas e em desenvolvimento, sendo considerada uma epidemia mundial que atinge indivíduos em todos os níveis socioeconômicos (TEICHMANN et al, 2006).

Pode ser definida como um acúmulo excessivo de gordura corporal, em extensão tal, que acarreta prejuízos à saúde, tais como alterações na função respiratória, distúrbios dermatológicos e do aparelho locomotor, além de favorecer o surgimento de enfermidades potencialmente letais, como hipertensão arterial sistêmica (HAS), intolerância a glicose, hipertrofia ventricular esquerda (HVE), hiperuricemia, elevação do fibrinogênio, síndrome metabólica (SM), síndrome da apneia obstrutiva do sono (SAOS) dislipidemias, doenças cardiovasculares, diabetes mellitus tipo 2 (DM2) e certos tipos de câncer (CA) (COUTINHO, 2007; PINHEIRO, FREITAS e CORSO, 2004; TAVARES et al, 2012; FELIX, SOARES e NÓBREGA, 2012).

Nos Estados Unidos (EUA), há mais de 15,0 milhões de indivíduos obesos graves (STURM, 2007), e no Brasil, 38,8 milhões de pessoas com 20 anos ou mais estavam acima do peso no ano de 2002 a 2003, sendo que $41,1 \%$ das pessoas do sexo masculino, encontraram-se acima do peso e $8,9 \%$ apresentaram-se obesas e, quanto ao sexo feminino, $40 \%$ estavam com sobrepeso e $13,1 \%$ com obesidade, totalizando uma média de $40,6 \%$ da população total do país acima do peso e 10,5 milhões de pessoas obesas (IBGE, 2004).

Opções clássicas, não cirúrgicas para conquistar perda de peso, em pacientes obesos, incluem restrição dietética, atividade física, modificações 
comportamentais, medicamentos e suporte psicológico, cujos resultados, frequentemente são limitados e obtidos em curtos períodos de tempo (SCHNEIDER e MUN, 2005).

Os insucessos dos tratamentos clínicos para a obesidade mórbida são superiores a 90\%, dessa forma, alternativas terapêuticas têm sido propostas, dentre as quais o tratamento cirúrgico é apontado como promissor para perda de peso significativa e duradoura (VALEZI et al., 2008). A cirurgia bariátrica (CB) como opção de tratamento, é indicada para pacientes com índice de massa corporal (IMC) a partir de $40 \mathrm{~kg} / \mathrm{m}^{2}$ (obesidade grave) ou acima de $35 \mathrm{~kg} / \mathrm{m}^{2}$ na presença de morbidades associadas como hipertensão, diabetes e apneia obstrutiva do sono (SANTOS et al., 2012).

A CB é realizada há pouco tempo no país e é um procedimento invasivo que pode causar algumas complicações advindas de sua implementação, sendo que o procedimento mais realizado tem sido a Derivação Gástrica em Y de Roux (DGYR) (ROCHA, 2012; SBCBM, 2006; LÓPEZ-FONTANA, FONERÓN e CASTILLO, 2012).

Conforme dados disponibilizados pelo Ministério da Saúde (MS) bem como, pela Sociedade Brasileira de Cirurgia Bariátrica e Metabólica (SBCBM), este procedimento vem sendo realizado em grande escala, tendo sido ampliada a sua realização tanto pelos serviços públicos de saúde quanto pela iniciativa privada, conforme demonstrada na tabela abaixo:

TABELA 01 - Frequência de cirurgias bariátricas realizadas no Brasil, entre os anos de 2003 à 2012, por fonte financiadora:

\begin{tabular}{lcc}
\hline Ano & Redes particular, suplementar e SUS & Rede pública (SUS) \\
\hline 2012 & $72.000^{\star \star \star \star}$ & 6.029 \\
2011 & $70.000^{\star \star \star}$ & 5.332 \\
2010 & $60.000^{\star \star}$ & 4.489 \\
2009 & $45.000^{*}$ & 3.731 \\
2008 & 38.000 & 3.139 \\
2007 & 33.000 & 2.974 \\
2006 & 29.500 & 2.528 \\
2005 & 22.000 & 2.266 \\
2004 & 18.000 & 1.872 \\
2003 & 16.000 & 1.778 \\
\hline Total & $\mathbf{4 0 3 . 5 0 0}$ & $\mathbf{3 4 . 1 3 8}$ \\
\hline
\end{tabular}

FONTE: Sociedade Brasileira de Cirurgia Bariátrica e Metabólica (SBCBM). Ministério da Saúde (MS).

* Destas 25\%foram realizadas por videolaparotomia. 
** Destas 35\% foram realizadas por videolaparotomia.

*** Destas $40 \%$ foram realizadas por videolaparotomia.

**** Destas $75 \%$ foram realizadas por videolaparotomia.

Um outro tipo de CB atualmente realizado é o de gastroplastia a Fobi-Capella, sendo que a taxa de mortalidade dessa cirurgia pode chegar até $1 \%$ nas grandes séries e as complicações gerais podem chegar até $40 \%$. Dentre as complicações verificadas podem ser citadas o surgimento de fístula da anastomose gastrojejunal, hematoma-seroma da incisão, hérnia incisional, embolia pulmonar, atelectasia e pneumonia pós-operatória, náuseas e vômitos persistentes, estenose da anastomose gastrojejunal, complicações com o anel, obstrução intestinal, síndrome de dumping, colecistite calculosa e complicações metabólicas como a deficiência de vitamina $\mathrm{B}_{12}$ e ferro (Fe) (ILIAS, 2011).

As técnicas cirúrgicas são divididas em restritivas, disabsortivas e mistas. Cada técnica promove alterações digestivas e absortivas características, necessitando de um programa educativo multidisciplinar exclusivo e direcionado tanto no pré quanto no pós-operatório, enfatizando os hábitos de atividade física e a necessidade de aderir às restritas recomendações dietéticas (RAVELLI et al, 2007).

As cirurgias que apresentam melhores resultados podem reduzir o peso entre 30 e $40 \%$ e esse efeito pode ser mantido por longos períodos (PAREJA, PILLA e GELONEZE, 2006). Assim, como todo procedimento cirúrgico especialmente do andar superior do abdome, a CB produz efeitos deletérios no sistema respiratório, como alterações na troca gasosa e na mecânica respiratória (PAZZIANOTTO-FORTI et al, 2012).

Indivíduos obesos submetidos à CB apresentaram acentuada redução tanto na frequência quanto na intensidade das dores musculoesqueléticas localizadas em ordem no quadril, tornozelo, pé e joelho (MELO e SÃO-PEDRO, 2012).

A complexidade desse procedimento é tamanha que a mesma transcende a vários âmbitos de estudo, como a sua realização em crianças e adolescentes, no tratamento ao DM, como o reganho de peso após a sua realização ou ainda, o surgimento de complicações de várias ordens junto ao paciente.

Dificuldades enfrentados na realização de procedimentos de intubação para realização da $C B$ são consagradas na literatura científica, em decorrência da apresentação de ordem morfológica do paciente (GEMPELERA, DíAZB e SARMIENTO, 2012). 
O tratamento cirúrgico da obesidade mórbida em adolescentes é aceito e realizado na maioria dos hospitais americanos e europeus e é o único método que mantem a perca de peso a longo prazo, melhorando as enfermidades associadas, a qualidade de vida e a autoestima do paciente (YESTE e CARRASCOSA, 2012).

Entretanto, numa recente publicação sobre a realização de $\mathrm{CB}$ em crianças e adolescentes, a mesma defende que esse procedimento é indicado unicamente em casos de obesidade extrema, em que foi observado fracasso em todo tratamento nutricional (ASTUDILLO e NEIRA, 2010).

Num recente ensaio clínico, foi possível concluir que a CB é eficaz para o controle do DM em pacientes obesos. Embora todas as técnicas cirúrgicas consigam bons resultados, estes estudos não teriam um tamanho amostral suficiente para demonstrar diferenças entre elas, necessitando de outros estudos que permitam concluir sua eficiência e eficácia (SCHAUER et al, 2012).

Outra questão relacionada a esse procedimento se refere ao aumento do peso, que ocorre mais frequentemente entre 03 e 06 anos depois da cirurgia. $O$ reganho de $25 \%$ do peso perdido previamente por efeito da CB tem sido considerado por alguns autores como um critério suficiente para realizar cirurgia revisional. Em outros estudos se tem considerado que o reganho de peso tem favorecido o reaparecimento de comorbidades com o DM (PAPAPIETRO, 2012).

Questões nutricionais também se configuram enquanto fenômenos resultantes da realização da CB. A deficiência na utilização de nutrientes pode ser considerada uma das desvantagens da DGYR, já que esta envolve o componente disabsortivo. Em função desse prejuízo anatômico, é importante enfatizar a necessidade de suplementação nutricional, a fim de garantir os macro e micronutrientes essenciais para o bom funcionamento do organismo e a importância e indispensabilidade do acompanhamento nutricional (MANIGLIA et al, 2012).

Fenômenos de fundo psíquico e emocional também são referidos na literatura científica em decorrência do desenvolvimento desse procedimento, como por exemplo, casos onde um incremento da agressividade é referido pelos pacientes após a cirurgia (LEAL e BALDIN, 2007), ou ainda, suicídio de pacientes previamente depressivos após a realização da CB em uma amostra de 16.683 pacientes operados na Pensilvânia (OMALU et al, 2005). A esse respeito, surge a necessidade de estudos investigando o impacto da $\mathrm{CB}$ sobre comorbidades psiquiátricas com atenção especial ao risco de suicídio (ADAMS et al. 2007). 


\section{OBJETIVOS DO ESTUDO:}

\section{OBJETIVO GERAL:}

* Analisar o conhecimento de estudantes universitários de uma instituição de ensino superior (IES), sobre a cirurgia bariátrica (CB).

\section{OBJETIVO ESPECÍFICO:}

* Analisar o perfil sociodemográfico de estudantes universitários de uma instituição de ensino superior (IES), participantes da presente pesquisa.

\section{METODOLOGIA:}

Trata-se de um estudo transversal, potencializado por uma dimensão quantitativa e que se propôs a analisar a questão do conhecimento de estudantes universitários da área da saúde, regularmente inscritos numa instituição de ensino superior (IES) sediada na cidade de Brasília, Distrito Federal (D.F.), sobre a cirurgia bariátrica $(\mathrm{CB})$.

Para aquisição de subsídios necessários a edificação do presente estudo, foi utilizado um instrumento de coleta de dados (ICD) proposto por Garrido Jr (2004), possuidor do título "Teste de entendimento aos pacientes candidatos à cirurgia da obesidade mórbida (TEPCCOM)", que permitiu analisar o conhecimento dos estudantes universitários/participantes deste estudo, sendo viável desta forma a aquisição das fontes primárias.

Após a aquisição dos dados, os mesmos foram organizados utilizando para tal atividade o software Microsoft Excel $2013 \AA$, pertencente ao pacote Microsoft Office $2013 \AA$, for Windows ${ }^{\circledR}$.

As fontes secundárias derivaram de artigos de periódicos científicos, produções acadêmicas e legislação correlata, adquirida após buscas bibliográficas eletrônicas junto à base de dados informatizados nacionais e internacionais (Cuiden $\AA$, Lilacs $\AA$, Medline $\AA$, Minerva-UFRJ $\AA$, Saber-USP ${ }^{\circledR}$, Scileo ${ }^{\circledR}$ ).

Foram utilizados os Descritores em Ciências da Saúde (DeCS) da Biblioteca Virtual da Saúde (BVS) sendo os mesmos, "Conhecimento" com o número de 
registro "33182" e identificador único "D019359", "Estudantes" com o número de registro "13721" e identificador único "D013334", "Peso Corporal" com o número de registro "1860" e identificador único "D001835“, "Obesidade" com o número de registro "9951" e identificador único "D009765", "Obesidade Mórbida" com o número de registro "19272" e identificador único "D009767", "Cirurgia Bariátrica" com o número de registro "51221" e identificador único "D050110".

Nas combinações e conjugação dos descritores em ciências da saúde selecionados, foram utilizados os operadores lógicos booleanos de pesquisa "and", "or" e "and not", permitindo desta forma potencializar o processo de identificação e aquisição de referências bibliográficas necessárias a edificação da presente pesquisa.

Objetivando respeitar integralmente os dispositivos expostos junto a resolução de número 466/2012 do Conselho Nacional de Saúde (CNS) do Ministério da Saúde (MS), relacionada às "diretrizes e normas regulamentadoras em pesquisa utilizando seres humanos", um projeto de pesquisa foi submetido à apreciação e avaliação bioética junto ao Comitê de Ética e Pesquisa (CEP) do Centro Universitário de Brasília (UNICEUB), sendo o mesmo aprovado com o número da CAAE "50679015.6.0000.0023".

Assim, todas as medidas protetivas foram tomadas integralmente, não havendo de nenhuma forma riscos aos sujeitos da pesquisa/participantes do presente estudo. O termo de conhecimento livre e esclarecido (TCLE) foi lido e explicado para todos os participantes da presente pesquisa que, após concordarem com o seu teor, assinaram o mesmo, respeitando desta forma o princípio bioético da autonomia.

Os autores do presente estudo conhecem e respeitam os dispositivos expostos junto à resolução de número 466/2012 do CNS do MS. O presente estudo, após concluída todas as etapas propostas, poderá ser apresentado junto a um congresso e eventos científico de âmbito nacional ou internacional, bem como, submetido a um periódico científico para sua publicação.

Se constituíram enquanto critérios de inclusão da presente pesquisa, estudantes universitários da área da saúde, tanto do sexo feminino quanto do sexo masculino, com idade igual ou superior a dezoito (18) anos, que estivessem regularmente inscritos junto a instituição de ensino superior (IES) participante da pesquisa, que estivessem regularmente inscrito junto ao primeiro período $\left(1^{\circ}\right)$ do ano 
de 2016, que estivessem regularmente inscritas junto ao período matutino e no noturno do ano de 2016, que possuíssem disponibilidade e quisessem participar livremente da presente pesquisa.

\section{RESULTADOS E DISCUSSÃO:}

Por meio do presente estudo foi permitido verificar que o perfil socioeconômico e demográfico dos graduandos em saúde participantes da presente pesquisa se constituiu de $48 \%(n=192)$ dos entrevistados declararam cursar enfermagem, 78,75\% ( $n=315)$ pessoas do sexo feminino, $87,75 \%(n=351)$ solteiras, $70,75 \%$ ( $n=283)$ não desenvolvem atividade remunerada, 93,25\% ( $n=373)$ negam enfermidade(s), 94,50\% ( $n=378)$ negam tabagismo, 78\% ( $n=312)$ negam consumo medicamentoso, 93\% ( $n=372)$ negam etilismo, 99,25\% ( $n=397)$ negam hipertensão arterial (HA), 100\% ( $n=400)$ negam diabetes mellitus (DM), 81,50\% $(n=326)$ negam sobrepeso e $95 \%(n=380)$ negam obesidade, conforme exposto junto a tabela de número 02.

TABELA 02 - Perfil socioeconômico e demográfico dos atores sociais participantes do presente estudo, por categorias analíticas, Brasília, Distrito Federal (D.F.), $(n=400)$.

\begin{tabular}{lcc}
\hline Categorias analíticas & Frequência & $\%$ \\
\hline Curso & & \\
\hline Enfermagem & 192 & 48,00 \\
Nutrição & 96 & 24,00 \\
Medicina & 62 & 15,50 \\
Biomedicina & 33 & 8,25 \\
Biologia & 17 & 4,25 \\
\hline Gênero & & \\
\hline Feminino & 315 & 78,75 \\
Masculino & 85 & 21,25 \\
\hline Estado civil & & \\
Solteira & 351 & 87,75 \\
Casado & 39 & 9,75 \\
União estável & 6 & 1,50 \\
Divorciada & 2 & 0,50 \\
Separada & 2 & 0,50 \\
\hline Realiza atividade remunerada & & \\
\hline Não & 283 & 70,75 \\
Sim & 116 & 29,00 \\
Não informado & 1 & 0,25 \\
\hline Possui enfermidade(s) & & \\
\hline
\end{tabular}




\begin{tabular}{ccc}
\hline Não & 373 & 93,25 \\
Sim & 27 & 6,75 \\
\hline É tabagista & & \\
Não & 378 & 94,50 \\
Sim & 22 & 5,50 \\
\hline Consume medicamentos & 312 & \\
\hline Não & 88 & 78,00 \\
Sim & & 22,00 \\
\hline É etilista & 372 & \\
\hline Não & 28 & 93,00 \\
Sim & & 7,00 \\
\hline Possui hipertensão arterial & 397 & \\
\hline Não & 3 & 99,25 \\
Sim & & 0,75 \\
\hline Se considera em sobrepeso & 326 & 81,50 \\
Não & 74 & 18,50 \\
Sim & & 95,00 \\
\hline Se considera obeso & 380 & 5,0 \\
\hline Não & 20 & $\mathbf{1 0 0 , 0 0}$ \\
Sim & $\mathbf{4 0 0}$ & \\
\hline Total & & \\
\hline
\end{tabular}

Fonte: Produção dos autores, 2016.

Já em relação as repostas disponibilizadas no que se refere aos questionamentos desenvolvidos, foi identificado reduzido conhecimento dentre os entrevistados, apenas na categoria analítica relacionada a necessidade de suplementação alimentar utilizando polivitamínicos após a realização da CB, conforme exposto junto a tabela de número 03.

TABELA 03 - Respostas aos questionamentos desenvolvidos, por frequência e percentual, Brasília, Distrito Federal (D.F.), Brasil, $(n=400)$ :

\section{Respostas aos questionamentos $\quad$ Frequência $\%$}

01 - Há outras cirurgias disponíveis para a obesidade mórbida além da indicada pelo meu cirurgião.

\begin{tabular}{lcc} 
Acertaram & 263 & 65,75 \\
Erraram & 124 & 31,00 \\
Não responderam & 13 & 3,25 \\
\hline 02 - O grampeamento do estômago e & as costuras intestinais nunca abrem e \\
resultam em infecções ou vazamentos. & 373 & 93,25 \\
Acertaram & 17 & 4,25 \\
Erraram & 10 & 2,50 \\
Não responderam & & \\
\hline 03 - Coágulos nas pernas ou pelves podem suceder da cirurgia da obesidade. & \\
Estes coágulos podem ser desprender e se encaminhar par os pulmões, causando \\
\hline
\end{tabular}




\begin{tabular}{|c|c|c|}
\hline \multicolumn{3}{|c|}{ uma sensação de falta de ar e dor no peito. } \\
\hline Acertaram & 313 & 78,25 \\
\hline Erraram & 78 & 19,50 \\
\hline Não responderam & 9 & 2,25 \\
\hline \multicolumn{3}{|c|}{$\begin{array}{l}04 \text { - Poucos meses após a cirurgia da obesidade, o paciente é capaz de comer e } \\
\text { beber qualquer coisa, que ele deseja em qualquer quantidade. }\end{array}$} \\
\hline Acertaram & 373 & 93,25 \\
\hline Erraram & 16 & 4,00 \\
\hline Não responderam & 11 & 2,75 \\
\hline \multicolumn{3}{|c|}{$\begin{array}{l}05 \text { - A cirurgia da obesidade garante ao paciente a perda permanente de peso } \\
\text { após essa cirurgia. }\end{array}$} \\
\hline Acertaram & 357 & 89,25 \\
\hline Erraram & 35 & 8,75 \\
\hline Não responderam & 8 & 2,00 \\
\hline \multicolumn{3}{|c|}{$\begin{array}{l}06 \text { - Diabetes, pressão alta, dores musculares ou doenças similares sempre } \\
\text { melhoram após essa cirurgia. }\end{array}$} \\
\hline Acertaram & 280 & 70,00 \\
\hline Erraram & 113 & 28,25 \\
\hline Não responderam & 7 & 1,75 \\
\hline \multicolumn{3}{|c|}{$\begin{array}{l}07 \text { - Existe a possibilidade de necessitar de cuidados intensivos no hospital (U.T. } \\
\text { I), por um tempo curto ou longo, após a cirurgia. }\end{array}$} \\
\hline Acertaram & 371 & 92,75 \\
\hline Erraram & 22 & 5,50 \\
\hline Não responderam & 7 & 1,75 \\
\hline \multicolumn{3}{|c|}{$\begin{array}{l}08 \text { - Algumas vezes a reoperação é necessária devido a sangramento, hérnias, } \\
\text { úlceras, abertura dos "grampos cirúrgicos", fístulas, obstrução dos intestinos ou } \\
\text { estômago e outras coisas. }\end{array}$} \\
\hline Acertaram & 360 & 90,00 \\
\hline Erraram & 33 & 8,25 \\
\hline Não responderam & 7 & 1,75 \\
\hline \multicolumn{3}{|c|}{$\begin{array}{l}09 \text { - Está operação para obesidade implica acompanhamento periódicos com } \\
\text { médico por toda a vida e outras cirurgias caso necessitar. }\end{array}$} \\
\hline Acertaram & 330 & 82,50 \\
\hline Erraram & 61 & 15,25 \\
\hline Não responderam & 9 & 2,25 \\
\hline \multicolumn{3}{|c|}{$\begin{array}{l}10 \text { - Após a cirurgia da obesidade, o paciente precisa tomar suplementos } \\
\text { vitamínicos por toda a vida. }\end{array}$} \\
\hline Acertaram & 196 & 49,00 \\
\hline Erraram & 197 & 49,25 \\
\hline Não responderam & 7 & 1,75 \\
\hline \multicolumn{3}{|c|}{$\begin{array}{l}11 \text { - Após esta cirurgia, nunca serie capaz de engolir (esmagado ou mastigado), os } \\
\text { comprimidos novamente. }\end{array}$} \\
\hline Acertaram & 375 & 93,75 \\
\hline Erraram & 14 & 3,50 \\
\hline Não responderam & 11 & 2,75 \\
\hline \multicolumn{3}{|c|}{12 - A cirurgia da obesidade não é um procedimento grande ou de risco. } \\
\hline Acertaram & 361 & 90,25 \\
\hline Erraram & 31 & 7,75 \\
\hline Não responderam & 8 & 2,00 \\
\hline
\end{tabular}




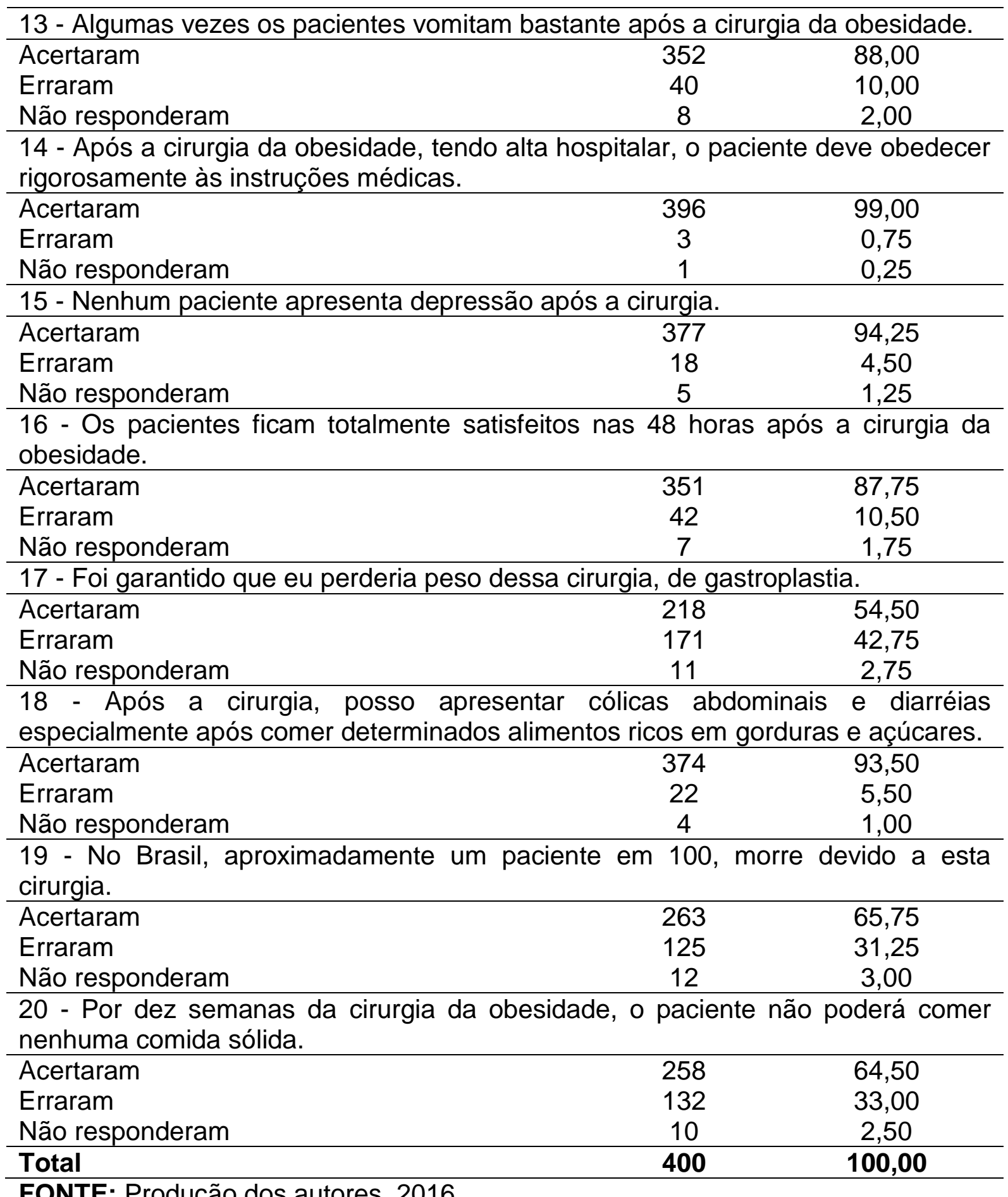

FONTE: Produção dos autores, 2016.

As deficiências nutricionais após realização da $\mathrm{CB}$, tem sido cada vez mais relatadas e analisadas junto à literatura científica, sendo muito frequentes, em razão da redução na ingestão oral e na absorção de nutrientes (MUSTAFA, 2014; TOREZAN, 2013; QUEIROZ, HAACK e MUNIZ, 2012; BORDALO, MOURÃO e 
BRESSAN, 2011; MECHANICK et al, 2008; KUSHNER, 2006; MALINOWSKI, 2006; BLOOMBERG et al, 2005; DEITEL e COWAN, 2000).

Todos os pacientes submetidos a CB devem receber suplementação de vitaminas e minerais diariamente. Entretanto, ainda não existe consenso em relação a melhor dose de suplementação dos micronutrientes (TOREZAN, 2013; MALINOWSKI, 2006).

Numa recente publicação, e apontado que as quantidades iniciais de suplementação de vitaminas/micronutrientes para normalizar os marcadores biológicos 2 anos após a realização da CB para pelo menos $3000 \mathrm{mg}$ de cálcio (Ca) com 7000 UI de vitamina D, 50.000 UI de vitamina A, 40 mg de zinco (Z), e de 200 $\mathrm{mg}$ de ferro (Fe) devem ser prescritos para começar (TOPARD et al, 2014).

Após a realização da $\mathrm{CB}$, existe a necessidade do uso de suplementação vitamínica diária por toda vida do paciente, sendo recomendados um (01) a dois (02) comprimidos de polivitamínico, via oral, diariamente (RAMOS e MELLO, 2015; QUEIROZ, HAACK e MUNIZ, 2012; BORDALO, MOURÃO e BRESSAN, 2011).

Alguns autores já sugerem doses adicionais além das doses presentes nos polivitamínicos usados de rotina, sendo o caso da vitamina B12, Fe, Z, Ca, da vitamina D, da vitamina A e da vitamina $E$ (TOREZAN, 2013; MALINOWSKI, 2006).

Entretanto a adesão a essa recomendação é variável, devido a fatores como o não reconhecimento da importância do uso destes, econômicos e a presença de múltiplas informações, algumas vezes conflituosas, a respeito da formula ideal de reposição (RAMOS e MELLO, 2015).

Estudos mostram que estas deficiências nutricionais são frequentes em pessoas que se encontrem gravemente obesas ou obesas mórbidas, mesmo antes do procedimento cirúrgico e que a CB pode agravar o quadro (CARLIN et al., 2006; YOSSEF et al., 2007).

As deficiências nutricionais no pós-operatório (PO) podem ser atribuídas a diversos fatores como por exemplo, a deficiência pré-cirúrgica, a ingestão alimentar reduzida, a suplementação inadequada, a má-absorção de nutrientes e a terapia nutrológica inadequada, como a reduzida monitorização ou ainda, segundo alguns autores, pela ausência de seguimento no PO (RAMOS e MELLO, 2015; RAVELLI et al, 2007; BLOOMBERG et al, 2005).

Nesse sentido, cada técnica da CB promove alterações digestivas e absortivas características, necessitando de um programa educativo multidisciplinar 
exclusivo e direcionado tanto no pré quanto no pós-operatório, enfatizando os hábitos de atividade física e a necessidade de aderir às restritas recomendações dietéticas (RAVELLI et al, 2007; BLOOMBERG et al, 2005; ALVAREZ-LEITE, 2004).

Nas pessoas submetidas à CB principalmente pelas técnicas de by-pass gástrico em Y-de-Roux (BGYR) ou a derivação biopancreática/ Duodenal Switch (DBP/DS) as mesmas, apresentam um maior risco em desenvolver deficiências nutricionais, devido, principalmente, à exclusão de partes do trato gastrointestinal, prejudicando a absorção eficiente dos nutrientes e evidenciando que, as técnicas cirúrgicas com características disabsortivas exercem um maior impacto na absorção de vitaminas e minerais e frequentemente resultam em deficiências nutricionais (RAMOS e MELLO, 2015; BORDALO, MOURÃO e BRESSAN, 2011).

As técnicas de CB do tipo disabsortivas são as que promovem maior incidência de deficiência dos micronutrientes, que é proporcional ao comprimento da área desviada e à porcentagem de perda de peso (MUSTAFA, 2014; QUEIROZ, HAACK e MUNIZ, 2012; BLOOMBERG et al, 2005; ALVAREZ-LEITE, 2004).

As cirurgias mistas com maior componente disabsortivo acarretam mais carências nutricionais do que aquelas com menor disabsorção (MUSTAFA, 2014).

Numa pesquisa desenvolvida na modalidade de estudo de caso clínico, realizado com uma paciente com 25 anos de idade e com diagnóstico de obesidade grau III que engravidou após três (03) meses de cirurgia, a mesma aponta para deficiências de alguns nutrientes como ferro (Fe), vitaminas do complexo B (B1, B2, B3, B5, B6, B7, B9, B12), vitaminas lipossolúveis, sendo necessária prescrição de suplementação objetivando reverte o quadro em questão (QUEIROZ, HAACK e MUNIZ, 2012).

Desta forma, após a realização da $\mathrm{CB}$, os hábitos alimentares do paciente sofrem uma série de mudanças significativas, surgindo desta forma, a necessidade do acompanhamento com uma equipe multiprofissional de saúde, fornecendo condições para que seja percebida a amplitude do processo ao qual o mesmo foi submetido para tratamento da obesidade ou obesidade mórbida (MATTOS et al, 2010; RAVELLI et al, 2007).

Após a realização da $\mathrm{CB}$ em suas várias modalidades, várias são as substâncias que apresentam carência junto ao organismo, sendo principalmente as vitaminas hidrossolúveis (vitamina B12, ácido fólico, vitamina B1), as vitaminas lipossolúveis (vitamina $D$, vitamina $A$, vitamina $E$, vitamina $K$ ), além da deficiência de 
elementos traço (ferro, cobre, zinco e selênio) (TOREZAN, 2013; QUEIROZ, HAACK e MUNIZ, 2012).

Já em outros estudos, a CB está associada a alterações no metabolismo ósseo, perda óssea e a um possível aumento de fraturas, sendo necessário realizar junto a este paciente, uma intervenção nutricional aliada à suplementação regular de vitamina D e cálcio ( $\mathrm{Ca}$ ), além de uma vida saudável (RAMOS e MELLO, 2015; MUSTAFA, 2014; TOREZAN, 2013; MALINOWSKI, 2006;).

As referidas deficiências nutricionais associadas às $C B$, são percebidas devido aos desvios desenvolvidos junto as estruturas intestinais, nos quais há inúmeros sítios absortivos de nutrientes (KUSHNER, 2006; BLOOMBERG et al, 2005; DEITEL e COWAN, 2000).

Devido à baixa ingestão alimentar por este paciente e após a realização da CB, o mesmo possui a necessidade de suplementação de vitaminas e de minerais o mais breve possível. Nesse sentido, a suplementação nutricional, geralmente, não se inicia logo após a $\mathrm{CB}$, devido às dificuldades verificadas em ingerir comprimidos e também, às intolerâncias causadas por eles (CRUZ e MORIMOTO, 2004; GARRIDO Jr et al, 2002; MACLEAN, RHODE e SHIZGAL, 1983).

As CB promovem ainda uma redução severa no consumo alimentar, o que acarreta a ingestão de dietas do tipo hipocalóricas e deficientes em micronutrientes, permitindo consequentes complicações nutricionais (MUSTAFA, 2014; TOREZAN, 2013; RAVELLI et al, 2007).

Objetivando prevenir o surgimento de complicações no PO de $\mathrm{CB}$, alguns apontamentos são propostos pela literatura científica corrente. O sucesso da CB a longo prazo passa pelo seguimento das orientações dietéticas, ou seja, aumentar a consistência dos alimentos gradualmente, ao longo de um período de 1 a 2 meses, avançando de líquidos para uma dieta leve e, finalmente, uma dieta regular com o objetivo de evitar deficiências nutricionais (LO MENZO, 2014).

Para alguns autores, o tratamento das deficiências nutricionais destes pacientes deve ser composto de megadoses de micronutrientes, levando-se em consideração a diminuída biodisponibilidade, menor ingestão e área de absorção decorrente do ato cirúrgico (FAÉ, LIBERALI e COUTINHO, 2015).

Já em outros estudos, devem ser prescritos multivitaminas e suplementação mineral, para tratamento da anemia no PO. Já realização de triagem de deficiências através de exames laboratoriais, individualizando algumas reposições, permite 
melhor adequação de suplementação ou tratamento de deficiências especificas (FAÉ, LIBERALI e COUTINHO, 2015; RAMOS e MELLO, 2015; KWON et al, 2014).

Desta forma é fundamental o acompanhamento clínico nutricional de todas as pessoas submetidas à $\mathrm{CB}$ a fim de garantir a manutenção da perda de peso de forma saudável (MUSTAFA, 2014; TOREZAN, 2013; BORDALO, MOURÃO e BRESSAN, 2011).

\section{CONSIDERAÇÕES FINAIS:}

Os objetivos traçados junto ao presente estudo foram alcançados integralmente, além da metodologia de pesquisa utilizada permitir a realização das atividades propostas com eficiência e qualidade.

Por meio do presente estudo foi possível verificar que os estudantes universitários entrevistados, possuem conhecimento no que se refere a $\mathrm{CB}$ em seus vários aspectos. Entretanto, especificidades relacionadas a este procedimento cirúrgico operatório, por exemplo, o consumo de polivitaminicos após a sua realização desta cirurgia, enquanto forma de suplementação alimentar, não se constitui enquanto questão de conhecimento pleno dos atores sociais participante da presente pesquisa.

Desta forma, uma das evidências verificadas na presente pesquisa foi a "poderosa" e "hegemônica" influência dos meios de comunicação, em todas as suas categorias e dimensões, permitindo nesse sentido, um maior conhecimento das questões relacionadas ao procedimento cirúrgico operatório de redução do peso corporal analisado.

A necessidade de redução e controle do peso corporal elevado de pessoas que se encontram obesas ou obesas mórbidas, além do combate e regularização de enfermidades e comorbidades relacionadas, ampliam o quantitativo de pessoas que aderem a esse tratamento cirúrgico, enquanto um dos últimos recursos para tratamento de doenças pré-existentes e reestabelecimento à saúde.

Entretanto, este procedimento cirúrgico delicado e que provocará grandes modificações junto a estrutura corporal da pessoa a ele submetido, não pode e nem deve ser indicado a pessoas que dele não necessitem verdadeiramente ou, que possuam interesses de ordem estética. 


\section{REFERÊNCIAS BIBLIOGRÁFICAS:}

ADAMS et al. Long-term mortality after gastric bypass surgery. $\mathrm{N}$ Engl $\mathrm{J}$ Med. 2007:357(8).753-761.

ALVAREZ-LEITE JI. Nutrient deficiencies secondary to bariatric surgery. Curr Opin Clin Nutr Metab Care. 2004;7(5):569-75.

ASTUDILLO, JJA, NEIRA, PA. Cirugía de la obesidad en niños y adolescentes. Rev Ecu. Ped. 2010:10(2).38-42.

BLOOMBERG RD, Fleishman A, Nalle JE, Herron DM, Kini S. Nutritional Deficiencies Following Bariatric Surgery: What Have We Learned?.Obes Surg 2005;15:145-54.

BORDALO, Lívia A.; MOURÃO, Denise Machado; BRESSAN, Josefina. Deficiências nutricionais após cirurgia bariátrica. Por que ocorrem? Acta Med Port 2011; 24(S4): 1021-1028.

CARLIN, AM; RAO, DS; MESLEMANI, AM; GENAW, JÁ: PARIKH, NJ: LEVY, S et al. Prevalence of vitamin $D$ depletion among morbidly obese patients seeking gastric bypass surgery. Surg Obes Relat Dis. 2006; 2 (2): 98-103.

COSTA, Dayanne da. Eficiência do acompanhamento nutricional no pré e pósoperatório da cirurgia bariátrica. Revista Brasileira de Obesidade, Nutrição e Emagrecimento, São Paulo v.7, n.39, p.57-68, Maio/Jun. 2013.

COUTINHO W. Etiologia da obesidade. Rev ABESO. [Internet]. 2007 [cited 2008 Dec 20]; 7(30):14 p. Available from: http://www.abeso.org.br/pdf/ Etiologia\%20e\%20Fisiopatologia\%20-\%20Walmir\%20Coutinho.pdf.

CRUZ, MRR; MORIMOTO, IMI. Intervenção nutricional no tratamento cirúrgico da obesidade mórbida: resultados de um protocolo diferenciado. Rev Nutrição 2004;17(2):263-72.

DEITEL, M; COWAN-Jr, GSM. Update: Surgery for the morbidly obese patient. Canadá: FD-Communications; 2000. p.161-70. 
FAÉ, Caroline; LIBERALI, Rafaela; COUTINHO, Vanessa Fernandes. Deficiência de nutrientes a longo prazo no pós-operatório de cirurgia bariátrica - revisão sistemática. SaBios: Rev. Saúde e Biol., v.10, n.2, p.46-53, mai./ago., 2015.

FELIX, LG. Soares, MJGO. Nóbrega, MML da. Protocolo de assistência de enfermagem ao paciente em pré e pós-operatório de cirurgia bariátrica. Rev Bras Enferm. 2012:65(1).83-91.

GARRIDO JR, AB; FERRAZ, EM; BARROSO, FL; MARCHESINI, JB; SZEGÖ, T. Cirurgia da obesidade. São Paulo: Atheneu; 2002, p.255-72.

GEMPELERA, FE. Díazb, L, Sarmiento, L. Manejo de la vía aérea em pacientes llevados a cirugía bariátrica en el Hospital Universitario de San Ignacio, Bogotá, Colombia. Rev Colomb Anestesiol. 2012:40(2):119-123.

ILIAS, EJ. Quais as complicações mais frequentes da gastroplastia a Fobi-Capella? Como tratá-las? Rev Assoc Med Bras. 2011:57(4).365-366.

INSTITUTO BRASILEIRO DE GEOGRAFIA E ESTATÍSTICA. Diretoria de Pesquisas. Coordenação de Índices de Preços. Pesquisa de Orçamentos Familiares 2002-2003. Disponível em: http://www.ibge.gov.br/home/estatistica/populacao/condicaodevida/pof/2002/default.s $\mathrm{htm}$

KUSHNER, RF. Micronutrient deficiencies and bariatric surgery. Curr Opin Endocinol Diab. 2006;13(5):405-11.

KWON, Y. et al. Anemia, iron and vitamin B 12 deficiencies after Sleeve gastrectomy compared to Roux-en-Y gastric bypass: a meta-analysis. Surgery for Obesity and Related Diseases, v. 10, n. 4, p. 589-597, 2014.

LEAL CW, Baldin N. O impacto emocional da cirurgia bariátrica em pacientes com obesidade mórbida. Rev Psiquiatr RS. 2007:29(3).324-7.

LO MENZO, E. et al. Nutritional Implications of Obesity: Before and After Bariatric Surgery. Bariatric Surgical Practice and Patient Care, v. 9, n. 1, p. 9-17, 2014.

LÓPEZ-FONTANA, G. Fonerón V., A. , Castillo C., AO. Nefrolitotomía percutánea en pacientes con cirugía bariátrica: ¿mayor riesgo de complicación? Rev. Chilena de Cirugía. 2012. 64(5).468-471.

MACLEAN, LD; RHODE, BM; SHIZGAL, HM. Nutrition Following Gastric Operations for Morbid Obesity. Ann Surg 1983;198(3):347-355

MALINOWSKI, SS. Nutritional and metabolic complications of bariatric surgery. Am J Med Sci. 2006; 331 (4): 219-225.

MANIGLIA, FP. et al. Alterações e adaptações hormonais relacionadas ao apetite no pós-operatório de cirurgia bariátrica. Rev Bras Nutr Clin. 2012.27(3).199-203. 
MATTOS, F.C.C.; FROSSARD, R.C.; COHEN, L.; ROSADO, E.L. Adesão e eficácia do acompanhamento nutricional no pré-operatório de pacientes submetidos a gastroplastia redutora em $\mathrm{Y}$ de Roux em um Hospital Federal Militar do Rio de Janeiro. Metabólica. Vol. 11. Núm. 1. 2010. p. 5-11.

MECHANICK, J. et al. Medical guidelines for clinical practice for the perioperative nutritional, metabolic, and nonsurgical support of the bariatric surgery patient. Endocr Pract. 2008; 4(1): 109-84.

MELO, IT de. São-Pedro, M. Dor musculoesquelética em membros inferiores de pacientes obesos antes e depois da cirurgia bariátrica. ABCD Arq Bras Cir Dig. 2012:25(1):29-32.

MUSTAFA, Sumaia. A cirurgia bariátrica e a perda óssea. International Journal of Nutrology, v.7, n.1, p. 22-30, Jan/Abr 2014.

OMALU BI, Cho P, Shakir AM, Agumadu UH, Rozin L, Kuller LH, et al. Suicides following bariatric surgery for the treatment of obesity. Surg Obes Relat Dis.2005:1(4).447-9.

PAPAPIETRO V, K. Reganancia de peso después de la cirugía bariátrica. Rev. Chilena de Cirugía. 2012:64(1). 83-87.

PAREJA JC, Pilla VF, Geloneze BN. Operational mechanisms of anti-obesity surgeries. Einstein. 2006;4(1):S120-S4.

PAZZIANOTTO-FORTI EM, et al. Aplicação da pressão positiva contínua nas vias aéreas em pacientes em pós-operatório de cirurgia bariátrica. Fisioter Pesq. 2012:19(1):14-19.

PINHEIRO ARO, Freitas SFT, Corso ACT. Uma abordagem epidemiológica da obesidade. Rev Nutr. 2004:17(4):523-33.

QUEIROZ, Cristiane de Souza; HAACK, Adriana; MUNIZ, Lidiane Batista. Caso Clínico de gestante com obesidade grau III submetida à cirurgia bariátrica. Revista de Divulgação Científica Sena Aires 2012; Julho-Dezembro (2): 163-170.

RAMOS, Camila Perlin; MELLO, Elza Daniel de. Manejo nutrológico no pósoperatório de cirurgia bariátrica. International Journal of Nutrology, v.8, n.2, p. 39-49, Mai / Ago 2015.

RAVELLI, Michele Novaes et al. Obesidade, cirurgia bariátrica e implicações nutricionais. RBPS 2007:20(4).p. 259-266.

ROCHA, JCG. Deficiência de Vitamina B12 no pós-operatório de Cirurgia Bariátrica. International Journal of Nutrology. 2012.5(2).82-89. 
SANTOS, MTA dos, el at. Alterações de parâmetros relacionados ao metabolismo ósseo em mulheres submetidas à derivação gástrica em $\mathrm{Y}$ de Roux. Arq Bras Endocrinol Metab. 2012.56(6). 376-82.

SCHAUER PR, et al. Bariatric surgery versus intensive medical therapy in obese patients with diabetes. N Engl J Med. 2012. 366(17):1567-76.

SCHNEIDER, BE; MUN, EC. Surgical management of morbid obesity. Diabetes Care. 2005:28(2).475-80.

SOCIEDADE BRASILEIRA DE CIRURGIA BARIÁTRICA E METABÓLICA. Consenso Brasileiro Multissocietário em cirurgia da obesidade. 2006. Disponível em: $<$ http://www.sbcb.org.br/associados.asp?menu=2>. Acesso em 12 de janeiro de 2012.

STURM R. Increases in morbid obesity in the USA:2000-2005. Public Health. 2007:121(7).492-496.

TAVARES, I da S. et al. Função diastólica do ventrículo esquerdo em obesos graves em pré-operatório para cirurgia bariátrica. Arq Bras Cardiol. 2012:98(4).300-306.

TEICHMANN L, Olinto MTA, Costa JSD, Ziegler D. Fatores de risco associados ao sobrepeso e à obesidade em mulheres de São Leopoldo, RS. Rev Bras Epidemiol. 2006:9(3).360-73.

TOPART, P. et al. Biliopancreatic diversion requires multiple vitamin and micronutrient adjustments within 2 years of surgery. Surgery for Obesity and Related Diseases, v. 10, n. 5, p. 936- 941, 2014

TOREZAN, Erika Franco Gaeti. Revisão das principais deficiências de micronutrientes no pós-operatório do Bypass Gástrico em Y de Roux. International Journal of Nutrology, 2013: v.6, n.1, p. 37-42, Jan/Abr.

VALEZI AC, et al. A importância do anel de silicone na derivação gástrica em Y-deRoux para o tratamento da obesidade. Rev Col Bras Cir. 2008:35(1).18-22.

YESTE D, Carrascosa A. El manejo de la obesidad en la infancia y adolescencia: de la dieta a la cirugía. Endocrinol Nutr. 2012. http://dx.doi.org/10.1016/j.endonu.2012.03.013.

YOUSSEF Y, Richards WO, Sekhar N, Kaiser J, Spagnoli A, Abumrad N et al. Risk of secondary hyperparathyroidism after laparoscopic gastric bypass surgery in obese women. Surg Endosc. 2007; 21 (8): 1393-1396.

WORLD HEALTH ORGANIZATION. (2000). Obesity: preventing and managing the global epidemic. Report of a WHO consultation. Geneva.

WORLD HEALTH ORGANIZATION. (2009). Obesity and overweight. Geneva. Adquirido em [http://www.who.int/mediacentre/factsheets/fs311/en/index.html]. 
ANEXO 01: Instrumento de Coleta de Dados (ICD) proposto por Garrido Jr (2004), possuidor do título "Teste de entendimento aos pacientes candidatos à cirurgia da obesidade mórbida (TEPCCOM)":

Todas as questões são de Certo/Errado. Por favor, marque com um " $x$ " somente na resposta que julgar correta.

01 - Há outras cirurgias disponíveis para a obesidade mórbida além da indicada pelo meu cirurgião.

Certo ( ) Errado ( )

02 - O grampeamento do estômago e as costuras intestinais nunca abrem e resultam em infecções ou vazamentos.

Certo ( ) Errado ( )

03 - Coágulos nas pernas ou pelves podem suceder da cirurgia da obesidade. Estes coágulos podem ser desprender e se encaminhar par os pulmões, causando uma sensação de falta de ar e dor no peito.

Certo ( ) Errado ( )

04 - Poucos meses após a cirurgia da obesidade, o paciente é capaz de comer e beber qualquer coisa, que ele deseja em qualquer quantidade.

Certo ( ) Errado ( )

05 - A cirurgia da obesidade garante ao paciente a perda permanente de peso após essa cirurgia.

Certo ( ) Errado ( )

06 - Diabetes, pressão alta, dores musculares ou doenças similares sempre melhoram após essa cirurgia.

Certo ( ) Errado ( )

07 - Existe a possibilidade de necessitar de cuidados intensivos no hospital (U.T.I.), por um tempo curto ou longo, após a cirurgia.

Certo ( ) Errado ( ) 
08 - Algumas vezes a reoperação é necessária devido a sangramento, hérnias, úlceras, abertura dos "grampos cirúrgicos", fístulas, obstrução dos intestinos ou estômago e outras coisas.

Certo ( ) Errado ( )

09 - Está operação para obesidade implica acompanhamento periódicos com médico por toda a vida e outras cirurgias caso necessitar.

Certo ( ) Errado ( )

10 - Após a cirurgia da obesidade, o paciente precisa tomar suplementos vitamínicos por toda a vida.

Certo ( ) Errado ( )

11 - Após esta cirurgia, nunca serie capaz de engolir (esmagado ou mastigado), os comprimidos novamente.

Certo ( ) Errado ( )

12 - A cirurgia da obesidade não é um procedimento grande ou de risco.

Certo ( ) Errado ( )

13 - Algumas vezes os pacientes vomitam bastante após a cirurgia da obesidade.

Certo ( ) Errado ( )

14 - Após a cirurgia da obesidade, tendo alta hospitalar, o paciente deve obedecer rigorosamente às instruções médicas.

Certo ( ) Errado ( )

15 - Nenhum paciente apresenta depressão após a cirurgia.

Certo ( ) Errado ( )

16 - Os pacientes ficam totalmente satisfeitos nas 48 horas após a cirurgia da obesidade.

Certo ( ) Errado ( )

17 - Foi garantido que eu perderia peso dessa cirurgia, de gastroplastia.

Certo ( ) Errado ( )

18 - Após a cirurgia, posso apresentar cólicas abdominais e diarréias especialmente após comer determinados alimentos ricos em gorduras e açúcares.

Certo ( ) Errado ( )

19 - No Brasil, aproximadamente um paciente em 100, morre devido a esta cirurgia.

Certo ( ) Errado ( )

20 - Por dez semanas da cirurgia da obesidade, o paciente não poderá comer nenhuma comida sólida.

Certo ( ) Errado ( ) 
ANEXO 02: Comprovante de submissão do projeto de pesquisa ao Comitê de Ética e Pesquisa do Centro Universitário de Brasília (CEP-UNICEUB): 


\section{CENTRO UNIVERSITÁRIO DE Plotoforma BRASÍLIA - UNICEUB}

\section{COMPROVANTE DE ENVIO DO PROJETO}

\section{DADOS DO PROJETO DE PESQUISA}

Título da Pesquisa: Conhecimento de estudantes universitários sobre a cirurgia bariátrica (CB).

Pesquisador: Linconl Agudo Oliveira Benito

Versão: 1

CAAE: 50679015.6 .0000 .0023

Instituição Proponente:

Centro Universitário de Brasilia - UNICEUB

\section{DADOS DO COMPROVANTE}

Número do Comprovante:

$111693 / 2015$

Patrocionador Principal:

Centro Universitário de Brasilia - UNICEUB

Informamos que o projeto Conhecimento de estudantes universitários sobre a cirurgia bariátrica (CB). que tem como pesquisador responsável Linconl Agudo Oliveira Benito, foi recebido para análise ética no CEP Centro Universitário de Brasilia - UNICEUB em 04/11/2015 às 11:15.

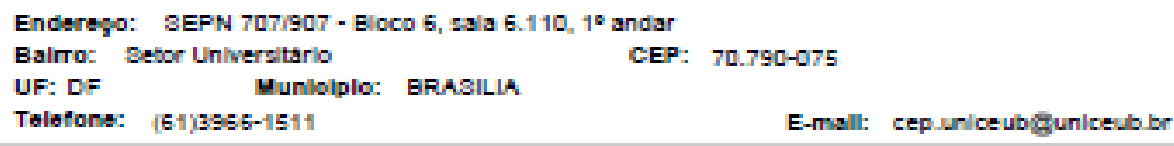

\section{AGRADECIMENTOS:}


A Deus em sua perfeição, onipotência, onisciência e onipresença, pela possibilidade diária de recomeçar e de avançar cotidianamente.

Agradecemos a Assessoria de Pós-Graduação e Pesquisa na pessoa da Prof. Dra. Fernanda Costa Vinhaes de Lima pela atenção e constante apoio na realização da presente pesquisa.

Ao Centro Universitário de Brasília (UNICEUB) pelo pioneirismo e iniciativa de disponibilização de uma bolsa de iniciação científica, sem a qual a presente pesquisa seria inviável. estudo.

E a você que direta ou indiretamente contribuiu para a conclusão do presente

Muito obrigado. 